\title{
La desindustrialización en Colombia desde la apertura económica*
}

\section{The des-industrialitation in Colombia}

Since economic aperture

\author{
Juan Carlos Buendía Pastrana** \\ Roberto Carlos Osorio Mass** \\ Jaime Eliécer Rangel Bolaños*** \\ Margarita Rosa Miranda Villera***
}

Recibido: 8 de abril de 2016

Revisado: 18 de mayo de 2016

Aprobado: 26 de mayo de 2016

* El artículo es el resultado de los procesos investigativos de los docentes en los programas de Administración de Empresas y Economía de las Universidad Pontificia Bolivariana Montería, Administración de Empresas de la Universidad Cooperativa de Colombia Montería y Administración de Empresas de la Corporación Unificada de Educación Superior. Cómo citar este artículo: Buendía Pastrana, J. C., Osorio Mass, R. O., Rangel Bolaños J. E. \& Miranda Villera, M. R. (2016). La desindustrialización en Colombia desde la apertura económica. Revista CIFE, 18(28), 71-89. (DOI: http://dx.doi.org/10.15332/s0124-3551.2016.0028.03

** Juan Carlos Buendía Pastrana y Roberto Carlos Osorio Mass tienen grado de máster en Dirección y Administración de Empresas (MBA), se desempeñan como docentes de la Corporacion unificada Nacional (CUN) y la Universidad Cooperativa de Colombia (UCG) respectivamente.). Correos electrónicos: juan_buendia@cun.edu.co

*** Jaime Rangel y Margarita Miranda son estudiantes de maestría de la Universidad del Norte. Jaime Rangel es docente de la Universidad Pontificia Bolivariana de Montería y Margarita Miranda trabaja como Asesora independiente. Estudiante de Maestría en Economía, Universidad del Norte,Correos electrónicos: jaime.rangelb@upb.edu.co 


\section{Resumen}

La desindustrialización en la economía colombiana ha generado una problemática en lo económico y social. Los factores más determinantes que se estudian son las evidencias y los síntomas del fenómeno de la enfermedad holandesa y el empleo. La economía colombiana presenta informalidad, esto ha llevado a problemas de competitividad y productividad en la industrial, puesto que el país es gran exportador de productos primarios que carecen de valor agregado, tales como petróleo, carbón, oro y minas. Este documento estudia los orígenes de la industria en Colombia, cuáles fueron los sectores que tuvieron mayor liderazgo y las coyunturas económicas internas y externas que condujeron al sector industrial a un proceso rápido de pérdida de participación dentro del PIB. Se analiza cada una de las variables que tienen relación con el sector industrial y cómo han sido afectadas desde la apertura económica; con base en dicho análisis, se darán unas conclusiones y unas posibles soluciones ante este fenómeno económico.

Palabras clave: Industrialización, política industrial, producción, estructura macroeconómica industrial.

Glasificación JEL: O14, L52, E23, L16

\section{Abstract}

The deindustrialization in the Colombian economy has generated a social and economic problem. The most determinant factors that were considered: evidences and symptoms of the phenomenon called "Dutch disease and employment". The Colombian economy the persistent informality has led to problems of competitiveness and productivity in industry, being as the country is a major exporter of primary products that with little or no none added value such as: oil, coal, gold and mines. This document examines when beginnings of Colombian industrial sector, which were the leading sectors and the internal and external economic conjunctures that led the industrial sector a rapid process of participation within the GDP. We analyze each of the variables that are related to the industrial sector and how they have been affected since the economic opening the conclusions and possible solutions to the economic phenomenon are found.

Keywords: Industrialization, industrial policy, production, industrial macroeconomic structure.

Classification JEL: O14, L52, E23, L16 


\section{Introducción}

En los últimos años, las economías a nivel mundial han presentado comportamientos en los cuales la participación del sector industrial dentro del producto interno bruto (PIB) ha venido disminuyendo. Desde la apertura de los mercados en los años ochenta y noventa a nivel global, el mundo se ha venido desindustrializando, siendo así un proceso normal y espontáneo para las economías más desarrolladas, en el que el PIB per cápita supera los diez mil dólares y cumple con un eslabón industrial avanzado y con gran contenido tecnológico, hasta tal punto que muchos recursos que antes eran de la industria se han ido desplazando a sectores más avanzados, como los servicios modernos.

Sin embargo, las economías en vía de desarrollo, precisamente como la economía colombiana, han presentado comportamientos contrarios a los países desarrollados. Partiendo de que ha existido una desindustrialización acelerada, muchos de los recursos del sector industrial se han desplazado hacia el sector primario y otros al sector del comercio, lo que ha generado efectos como síntomas de enfermedad holandesa, en los que se ha visto un incremento del sector minero-energético por parte de la economía colombiana en los últimos 20 años y que ha traído consecuencias en el sector industrial, tales como la revaluación del peso frente al dólar, hecho que afecta notoriamente las exportaciones industriales y es causante de problemas de competitividad y productividad en el sector.

Por esta razón, se analizarán variables que tienen relación con el sector industrial y cómo han sido afectadas desde la apertura económica; a partir de ello se darán unas conclusiones y unas posibles soluciones ante este fenómeno económico.

\section{Diseño metodológico}

Esta investigación se realizó bajo un enfoque descriptivo en el que la información utilizada para abordar la temática es de carácter secundario. En primer lugar, se analizarán estudios y evidencias de cómo ha sido el comportamiento de la industria a lo largo del período de estudio (1970-2013) y cuál ha sido la participación de la industria como aporte al PIB; de igual modo, se analizará qué variables han afectado directamente el sector industrial colombiano (productividad, competitividad, base productiva exportadora, capacidad para importar, diversificación productiva, comportamiento del sector primario y comportamiento del sector terciario).

Seguidamente, se buscará establecer cómo ha sido el comportamiento de la industria y del PIB desde los años noventa, lo que permitirá obtener una primera aproximación al objetivo principal de esta investigación, que se refiere a si existe o no un proceso de desindustrialización en Colombia. Con miras a alcanzar este objetivo, también se tomará como base la información publicada por la Comisión Económica para América Latina 
y el Caribe (GEPAL) y publicaciones de algunos autores que han tratado este tema, para así poder establecer cuáles han sido las variables más relevantes de este fenómeno y cómo se han comportado a lo largo del tiempo.

Es importante saber qué causas y qué consecuencias ha dejado el fenómeno de la desindustrialización en la economía colombiana, cuáles son las principales variables de este sector que mayormente han sido afectadas y qué mecanismos se han implementado para contrarrestar dicha problemática. De igual modo, es pertinente conocer de manera histórica, a través del tiempo, cómo se generó la industria en Colombia, para poder entender su comportamiento hoy.

\section{Proceso de desindustrialización en Colombia desde la apertura económica}

La producción de la industria manufacturera corresponde al sector secundario de la economía; se basa en la transformación continua y a gran escala de materias primas en productos transformables y tangibles, con procesos de trasformación controlada, sin interferencia o dependencia exterior al propio proceso productivo, y es la que mayor efecto multiplicador tiene sobre otras actividades de la economía colombiana. Esta industria se caracteriza principalmente por incluir actividades de transformación primaria o artesanal que no es continua ni a gran escala, y actividades terciarias que no transforman materialmente los productos.

Se dice que este sector es considerado efecto multiplicador, ya que arrastra de manera eficiente los diferentes sectores de la economía, principalmente el sector primario y el terciario; aparte de esto, ayuda a dinamizar variables como el empleo y a dinamizar toda la economía en general. A pesar de las bondades que genera el sector industrial dentro de una economía, durante las últimas dos décadas se ha visto una disminución considerable y acelerada de su participacióncon respecto al PIB frente a otros sectores de la economía.

Durante la segunda mitad del siglo XX, más exactamente a finales de siglo, se pudo evidenciar cómo la industria colombiana fue estancándose y rezagándose debido a diferentes factores económicos que la llevaron a tener una menor contribución en la contabilización del PIB y de su valor agregado. Como Clavijo, Vera y Fandiño (2012) mencionan, se estima que la relación entre producción industrial y producto interno bruto ha estado entre un $9 \%$ y $12 \%$ desde la apertura economía hasta nuestros días. 
La industria colombiana se ha venido desacelerando debido a factores múltiples como el fenómeno de la enfermedad holandesa de las exportaciones minero-energéticas, que representan el $24 \%$ de la producción nacional (Clavijo et al., 2012).

El incremento de la competencia de empresas internacionales llegadas al país después de la apertura económica ha traído consigo las dificultades que se presentan dentro del territorio colombiano para proveer a la población de servicios tales como energía y electricidad.

La CEPAL, a la cabeza de Raúl Prebish, es una institución que tenía como fin crear un modelo teórico, conceptual y estructural para la constitución del desarrollo de los países latinoamericanos (Naciones Unidas, 1985). En la década de los cincuenta, se planteó el concepto de sustitución de importaciones, teniendo como objetivo la modernización y el desarrollo industrial. Este modelo consistió en la formalización y evolución de la industria en tres etapas fundamentales:

- Industrialización por sustitución de importaciones de bienes livianos (1930- 1945): Esta primera fase del proceso de industrialización consistía en la creación de manufacturas simples con bajo desarrollo tecnológico que elaborasen bienes livianos para el consumo como, bebidas, alimentos, cervecerías, y productos agroindustriales, tabaco, etc. (FitzGerald, 1998).

- Industrialización por sustitución de importación de bienes intermedios y de consumo duradero (1945-1967): Hacia mediados de los años cincuenta, Colombia presentó un cambio estructural en la industria manufacturera, y así se dio la consolidación de la segunda etapa del modelo. Esta fase consistía en el fortalecimiento y avance tecnológico de la industria intermedia, precedidos del aumento significativo de acumulación de capital para la elaboración y comercialización de bienes intermedios, como textiles, algodón, caucho, cuero, trigo, acero, hierro. A finales de los años setenta, la diversificación industrial se centró en bienes de consumo tardíos, como papel, productos químicos derivados del petróleo, carbón, metales básicos e industria metalmecánica (Ocampo, Bernal, Avella , \& Errázuriz, 1987). Para esta segunda fase se registró un crecimiento económico en un orden del $6,4 \%$.

- Industrialización por sustitución de importaciones de bienes pesados o capital: En esta última fase de industrialización, las industrias debían poseer un avance tecnológico significativo y de innovación para la implementación ycreación de maquinaria pesada, esto con el objeto de tener acceso a una mayor acumulación de capital, consolidando así el modelo (ISI) (FitzGerald, 1998).

Con estas estrategias se esperaba que las economías latinoamericanas, especialmente la de Colombia, no dependieran más de los países con economías más desarrolladas 
importando sus productos, especialmente del sector industrial, sino que ya pudieran tener su propia autonomía y producir internamente los nuevos productos con la industria naciente, teniendo como meta la implementación gradual de las etapas del desarrollo industrial propuestas por la GEPAL y con ayuda del proteccionismo estatal. Sin embargo, en Colombia solamente se logró alcanzar las dos primeras etapas del modelo de sustitución de importaciones, pues las coyunturas del mercado en los órdenes nacional e internacional no permitieron desarrollar eficientemente la tercera etapa, que consistía en el desarrollo y el avance tecnológico de bienes de capital.

Colombia logró consolidar la primera etapa, en la cual pudo producir internamente lo que antes tenía que importar a los demás países en bienes livianos, es decir, manufacturas como cerámica, bebidas, cervecerías, alimentos semielaborados etc. El otro de los principales logros fue que consolidó una política económica exportadora por medio de la cual intensificó su diversificación productiva y pudo tener buena canasta de bienes, principalmente livianos, los cuales podrían ser exportados y comercializados a diferentes partes del mundo. Todo este proceso de industrialización que se llevó a cabo de 1930 a 1990 en Colombia tuvo grandes éxitos para la consolidación de la industria, como los anteriormente mencionados, pero también implicó tener períodos de estancamiento fuerte que lo dirigirían una desaceleración de la actividad económica.

Realizando un recorrido histórico de la industria colombiana desde la época de los años setenta, pasando por la apertura económica en 1990, hasta nuestros días, se puede decir que hacia los años setetenta y ochenta, Colombia presentó un estancamiento del sector industrial. Aunque para ese momento la industria reflejaba un $23 \%$ del PIB, se mostraron a grandes rasgos factores internos y externos que lo llevaron a su debilitamiento. Entre los factores internos se dieron fenómenos como la enfermedad holandesa, debido a la excesiva llegada de divisas provenientes del café, al manejo de políticas macroeconómicas y a la estrechez del mercado interno. Acerca de los externos, la difícil situación que se presentó en el mundo con la crisis petrolera que se dio en 1973, y que golpeó a toda la economía mundial, derivó de un shock de oferta agregada que propiciaron los países exportadores de petróleo en el Medio Oriente, por consiguiente, muchos países presentaron una disminución en cuanto a su crecimiento económico, y Colombia no fue la excepción. A partir de este hecho, el sector industrial empezó a tener poca participación en el PIB y, por ende, poco liderazgo en la economía.

En los años noventa se dio un fenómeno económico en el que el modelo anterior que se tenía, de protección y de sustitución, cambió por el de importaciones, llegando así la apertura económica o sistema económico neoliberal. Este nuevo modelo sostiene el libre comercio y la libre entrada de competencia extranjera al país. Fue entonces cuando la industria colombiana empezó a competir con las nuevas empresas extranjeras que llegaron a Colombia, y como consecuencias se dieron la salida del mercado de 
algunas empresas por no ser competitivas frente a las otras y la consolidación de la desindustrialización.

Por otra parte, se ha visto que las industrias se han venido debilitando y rezagando aceleradamente desde la apertura económica hasta nuestros días como consecuencia de la reducción de capacidad instalada industrial, que ha dado origen a que la productividad y la competitividad de la mano de obra se traspasen a otros sectores, como el incremento sustancial del sector terciario de la economía: finanzas, servicios (outsourcing) y trasporte, que han tenido una mayor participación en el PIB; esto se ha dado principalmente en las ciudades en donde se ha concentrado la mayor industria, especialmente en Bogotá, Medellín y Cali (Galvis, 2014).

Debido a la enfermedad holandesa, la desindustrialización se presenta con más fuerza en los países que fundamentan su crecimiento económico con base en las exportaciones de materias primas. En la enfermedad holandesa, la excesiva entrada de divisas a la economía nacional por medio de las exportaciones de commodities o materias primas tiende a revaluar la moneda, y esto trae como consecuencia una caída de las exportaciones, principalmente las agroindustriales.

Sin embargo, el tema de la desindustrialización en Colombia ha sido muy debatido en los últimos años. Algunos afirman que el problema es grave y que va a traer consecuencias devastadoras para la economía, pero otros dicen que no es un problema de gravedad, aunque sí de mucho cuidado, y que es tema de estudio, mientras que otros consideran que no hay dicha desindustrialización, sino que, por el contrario, ha evolucionado y ha hecho frente a la nueva industria mundial del siglo XXI; quienes comparten este último punto de vista sostienen que la industria y la manufactura han generado un cambio en la cadena de producción industrial como resultado de la desintegración vertical y también han provocado un traspaso de los medios de producción a otros sectores ¿Será esto posible? ¿Existirá en Colombia una desindustrialización o, por el contrario, está evolucionando de la industria moderna a la posmoderna? ¿Acaso será que el país se está primarizando, tal como lo hizo a mediados del siglo XIX, y estaremos retrocediendo en el tiempo? Estas son preguntas que se solucionarán a lo largo de la investigación.

Si bien el fenómeno de la desindustrialización es un aspecto económico que se ha desarrollado en todo el mundo y es un tema reciente, de no menos de 20 o 30 años, es importante establecer en qué condiciones se aborda. Debido a que es un tema nuevo y actual, es pertinente considerar que no ha llegado a una profunda madurez, en la que se hayan creado teorías consistentes y verídicas que confirmen este proceso. Sin embargo, se han hecho muchas investigaciones y estudios que han dado como resultado marcos conceptuales y modelos teóricos que se toman como referencias para abarcar el fenómeno de la desindustrialización. 
Carranza y Moreno (2013) realizaron un análisis del tamaño de la cadena de producción industrial colombiana y su componente de valor agregado con relación a la participación que este tiene en el PIB, partiendo del concepto de la trasformación productiva de la actividad industrial desde la apertura económica y de cómo esta trasformación ha atraído otros sectores de la economía de forma dinámica. Para la década de los noventa, la cadena de producción industrial dejó de crecer, pero a partir del año 2000 se evidenció que dicha producción creció persistentemente y que tuvo un papel representativo en la producción total de la economía. En esta investigación se muestra que las pequeñas y grandes industrias han tenido un cambio de localización, lo cual se evidencia en una desintegración vertical que ha causado un descenso de la participación del valor agregado industrial en el valor de la cadena productiva y en la manera como esta desintegración ha hecho que las industrias reduzcan su capacidad instalada, de tal forma que los recursos que se tenían antes se han ido desplazando al sector terciario de la economía nacional.

El objetivo de esta investigación era mostrar que la cadena de producción industrial no ha disminuido ni tampoco ha perdido participación en el PIB, sino que, a partir de los años noventa, la industria colombiana ha venido mostrando cambios en el tamaño y estructura de producción. Este trabajo sustenta y sugiere que en la industria se ha venido evidenciando un desmonte de la estructura vertical (desintegración vertical) y que finalmente alguna producción se ha ido desplazando al sector terciario, aumentando los servicios y el sistema financiero en el PIB. También afirma que existe inconveniencia en cuanto al uso del valor agregado como medida del tamaño de la industria, a diferencia de la medida focal del tamaño de la industria en otros países donde la medición de la industria manufacturera es el empleo industrial (Carranza y Moreno, 2013).

Finalmente, el crecimiento industrial desde los años noventa arroja resultados a partir de los cuales se infiere se han ido trasformando y desplazando hacia los diferentes sectores de la economía, especialmente al sector terciario, generando así sistemas de apoyo para la industria, que atienden sus necesidades de modo descentralizado. Por consiguiente, según la investigación de Carranza y Moreno (2013), los nuevos servicios nacientes para 1994 se contabilizaban para el sector industrial, pero ya para el año 2005 esa contabilización se incorporó en las actividades del sector terciario de la economía, generando así una disminución de la participación de la industria con respecto al PIB. Los autores afirman que no existe dicha desindustrialización, sino que el tamaño del sector industrial ha cambiado en su estructura vertical y que, de igual modo, se ha ido representando una tercerización de la economía, que se ha expandido al sector servicios, es decir, que la parte del valor agregado industrial hecho en 1994 se reclasificó en el sector servicios para el año 2005 como valor agregado en el sector servicios (Carranza y Moreno, 2013). 
Por su parte, Clavijo et al. (2012) se centran en demostrar las variables macroeconómicas que han llevado a Colombia a una desindustrialización desde 1965, pasando por la apertura económica hasta nuestros días. Los autores manifiestan que la principal causa de dicha desindustrialización ha sido ocasionada, a grandes rasgos, por la enfermedad holandesa. Hacia finales de la década de los sesenta y principios de los años setenta se evidenció cómo la industria quedó estancada por múltiples factores, pero el más representativo que se dio fue el de la enfermedad holandesa, proveniente del auge exportador del café, y que trajo como consecuencia un incremento sustancial de las exportación de commodities, el cual llevó a que se revaluara el tipo de cambio de la moneda colombiana, y esto hizo que las exportaciones cayeran en detrimento debido a los términos de intercambio. Aunque para ese tiempo el valor de la producción industrial y el valor agregado para el PIB eran del $23 \%$, se puede ver que, de ese tiempo hasta nuestros días, se ha venido desindustrializando aceleradamente con respecto a las industrias del mundo desarrollado.

Ya para los años ochenta y los noventa, pasando por la apertura económica, la industria colombiana sufrió una fuerte caída, acelerada como consecuencia del auge mineroenergético. Debido a la excesiva entrada de divisas al país, el sector industrial resultó fuertemente golpeado, principalmente en las exportaciones, hecho que generó un efecto dominó que afectó otras variables de la economía - como la utilidad, el empleo y la productividad- ${ }^{-}$provocó serias dificultades para proveer servicios básicos tales como telecomunicaciones, vías de trasporte, electricidad, un creciente encarecimiento relativo de la mano de obra y una marcada apreciación de la tasa de cambio (Clavijo et al., 2012).

La economía colombiana en los últimos tiempos se ha basado en las exportaciones de materias primas, esto ha hecho que los aportes al sector industrial caigan drásticamente debido a que no son competitivos en el mercado mundial. Es por esto que se postulan diferentes soluciones para evitar la creciente aceleración de la poca participación industrial; entre tales soluciones están la posibilidad de modernizar la infraestructura de trasporte, para así abaratar los costos de exportación e importación de productos diferentes de los commodities (compensando la apreciación cambiaria), y crear reformas laborales que permitan tener mano de obra calificada competitiva internacionalmente para reducir así los costos laborales unitarios (Clavijo et al., 2012).

Según la Asociación Nacional de Instituciones Financieras (ANIF), Colombia se ha venido desindustrializando desde 1975 hasta hoy, debido al bajo valor agregado que tienen las empresas industriales y de servicios. Para los años setenta, la contribución del sector industrial al PIB era del $24 \%$; actualmente, la industria colombiana se encuentra alrededor de un $12 \%$ de su participación y se estima que, para el período de comprende de 2015 a 2020, la participación de este sector oscile entre el $11 \%$ y el $9 \%$. Este efecto de desindustrialización se ve reflejado en el empleo, de manera que se plantea el reto, para todos estos años en Colombia, de invertir en ciencia y tecnología, principalmente en las universidades que propongan proyectos de innovación y competitividad, también 
invertir en el sector terciario de la economía, ya que estos se han denominado servicios modernos con alto nivel tecnológico (Clavijo et al., 2012).

Según Sanabria-Gómez (2007), la industrialización en Colombia se divide en tres etapas: la primera consiste en la consolidación del desarrollo industrial y de las políticas económicas que sirvieron de sustento hasta los años sesenta; la segunda, en el papel fundamental que jugó el Estado para el desarrollo industrial; y la tercera y más importante etapa se refiere a las tres últimas décadas de desindustrialización, en la que se busca evidenciar cómo el sector industrial ha venido teniendo una baja participación en las exportaciones y, por consiguiente, en la actividad económica.

El autor sugiere que una de las principales características de la desindustrialización es la productividad laboral, en la cual tomó como base dos períodos: el primero comprende de 1975 hasta 1990 y el segundo de 1990 hasta 2003. En el primer período, la industria colombiana tuvo una gran representación en el PIB, esto se deriva de que el Estado tenía políticas proteccionistas que evitaban la entrada de industrias extranjeras al país, lo cual generó que la mano de obra fuera intensiva en la industria y que la productividad laboral no fuera determinante en el desarrollo industrial. Para el segundo período, la industria colombiana se fue desacelerando como consecuencia de la nueva competencia mundial, que dejó como resultado una baja productividad laboral y baja competitividad entre las industrias colombianas y la industria mundial (Sanabria-Gómez, 2007).

Echavarría y Villamizar (2006) manifiestan que la desindustrialización está ligada principalmente a la productividad y a la competitividad. La base productiva de la industria está sujeta a la intensidad de mano de obra industrial no calificada, que no le permite obtener buenos resultados en la competencia industrial internacional. Los autores sostienen que la productividad va de la mano con la creación de valor agregado de la industria. En la medida en que en las industrias exista creación de valor agregado, la industria será más productiva.

Por su parte, Steiner (2011) muestra qué tanta participación ha perdido la industria mundial dentro del PIB al relacionarlo con distintos sectores de la economía. Este estudio sugiere que a nivel mundial este fenómeno es un proceso espontáneo, principalmente en las economías desarrolladas, en donde grandes recursos que la industria tenía en sus manos se han ido desplazando al sector terciario de la economía con gran contenido tecnológico, generando así un aumento en el sector de servicios. En Latinoamérica se evidencia un proceso semejante al de los países desarrollados, por ende, esta investigación sugiere cuál ha sido el comportamiento en Latinoamérica, y más exactamente en Colombia, con este fenómeno. Primeramente, se van a mostrar unas cifras de los distintos sectores en Colombia en las últimas cuatro décadas, ya que es importante evidenciar cómo las empresas industriales han venido rezagándose en los últimos años. 
Tabla 1. Participación del PIB sectorial en Colombia

\begin{tabular}{|c|c|c|c|c|c|c|}
\hline Sector/Año & Agricultura & Minería & Industria & Construcción & Comercio & Servicios \\
\hline $1970-1979$ & 18,6 & 1,7 & 20,3 & 7,2 & 15,3 & 36,9 \\
\hline $1980-1989$ & 17,2 & 2,3 & 18,9 & 8 & 14,3 & 39,3 \\
\hline $1990-1999$ & 16 & 4,4 & 16,6 & 6,2 & 12,9 & 43,4 \\
\hline $2000-2010$ & 14,1 & 5 & 15,8 & 5,8 & 11,9 & 47,4 \\
\hline
\end{tabular}

Fuente: Steiner (2011), ¿Se está “des-industrializando” Colombia? Pag. 16.

Se puede ver que, con el transcurso de los años, el sector industrial ha venido desacelerándose continuamente, a diferencia de lo que ocurre con el sector primario y el terciario de la economía, que tienen una participación cada vez más alta en el PIB. Esto es consecuencia del auge minero-energético que ha experimentado Colombia, de lo que se puede inferir que se están presentando síntomas de enfermedad holandesa. Durante los últimos 14 años, Colombia ha experimentado y atraído inversión extranjera directa, principalmente en la extracción de minería e hidrocarburos del sector primario de la economía colombiana, por ende, existe un aumento significativo del sector minero en las dos últimas décadas (Steiner, 2011).

Muchos autores afirman que este fenómeno económico se ha generado en el mundo en todas las economías. Como lo muestran las cifras de Fedesarrollo, existe un aumento de la participación del sector terciario dentro del PIB.

Tabla 2. Participación del valor agregado del sector terciario en el PIB a nivel mundial

\begin{tabular}{|c|c|c|c|c|c|c|c|}
\hline País/Año & Brasil & Chile & Golombia & México & Perú & A/L & Mundo \\
\hline $1970-1979$ & 48 & 52,4 & 45,6 & 56,2 & 47,7 & 50,6 & 54,5 \\
\hline $1980-1989$ & 45 & 53,8 & 46,9 & 57 & 57,4 & 51,1 & 58,9 \\
\hline $1990-1999$ & 60,5 & 53,8 & 52,3 & 65,8 & 61,8 & 60,8 & 64,8 \\
\hline $2000-2010$ & 66 & 53,8 & 59,5 & 63,6 & 59,2 & 62,2 & 68,9 \\
\hline
\end{tabular}

Fuente: Steiner (2011), ¿Se está “des-industrializando” Colombia? Pag. 17.

Estas cifras reflejan el comportamiento de la participación del sector terciario de la economía en los distintos países, aunque se hace hincapié en América Latina. Este fenómeno es un proceso normal que todos los países han experimentado de desarrollo, procesos productivos que antes estaban principalmente en el aparato industrial y pasaron al sector de servicios. Otra variable que es importante destacar es el comportamiento del valor agregado dentro de la industria. Al respecto, en los últimos 
ISSN: 0124-3551 / Año 18, No 28 / enero-junio / pp. 71-89

40 años se ha podido ver cómo este factor, que es relevante dentro de este sector, ha disminuido considerablemente.

En la tabla 3 se muestra el comportamiento del valor agregado dentro de la región latinoamericana: Colombia es uno de los países con menor tasa de valor agregado frente al global.

Tabla 3. Valor agregado de la industria en la región

\begin{tabular}{|c|c|c|c|c|c|c|}
\hline País/ Año & Brasil & Ghile & Colombia & México & Perú & A/L \\
\hline $1970-1979$ & 30 & 24,2 & 23 & 22,7 & 21,2 & 26,5 \\
\hline $1980-1989$ & 32,7 & 19,9 & 22,2 & 23,1 & 26,7 & 26,7 \\
\hline $1990-1999$ & 20,3 & 19,2 & 17,4 & 20,5 & 16,9 & 20 \\
\hline $2000-2010$ & 17,1 & 16,4 & 15,4 & 18,6 & 15,8 & 18 \\
\hline
\end{tabular}

Fuente: Steiner (2011), ¿Se está “des-industrializando" Colombia? Pag. 17.

En estas últimas cuatro décadas se ha evidenciado que la disminución del valor agregado en la industria manufacturera se ha acelerado, principalmente en Brasil.

Tabla 4. Subsectores de la industria colombiana y el promedio

\begin{tabular}{|c|c|c|c|c|c|}
\hline Sector/año & 1970-1979 & 1980-1989 & 1990-1999 & $2000-2010$ & Promedio \\
\hline Alimentos & $6,40 \%$ & $1,80 \%$ & $-0,50 \%$ & $2,60 \%$ & $2,60 \%$ \\
\hline Bebidas y tabaco & $7,50 \%$ & $2,00 \%$ & $-0,20 \%$ & $2,80 \%$ & $3 \%$ \\
\hline Textiles y calzado & $6,20 \%$ & $1,80 \%$ & $-3,20 \%$ & $5,90 \%$ & $2,70 \%$ \\
\hline Madera papel y otros & $7,20 \%$ & $4,00 \%$ & $1,30 \%$ & $5 \%$ & $4,40 \%$ \\
\hline Refinado de petróleo & $2,80 \%$ & $6,60 \%$ & $1,80 \%$ & $1,60 \%$ & $3,20 \%$ \\
\hline Químicos y metales & $6,60 \%$ & $3,50 \%$ & $0,90 \%$ & $5,60 \%$ & $4,10 \%$ \\
\hline Maquinaria y equipo & $12,50 \%$ & $2,90 \%$ & $-0,10 \%$ & $8,00 \%$ & $5,80 \%$ \\
\hline PIB industrial & $6,60 \%$ & $2,70 \%$ & $0,10 \%$ & $4,30 \%$ & $3,40 \%$ \\
\hline
\end{tabular}


Estas cifras arrojan en promedio la participación de cada subsector perteneciente a la industria con respecto al PIB industrial. Como se puede observar, en la década de los noventa, desde la apertura, han existido crecimientos negativos de algunos sectores de la industria, como el alimenticio, el de bebidas, tabaco, maquinaria y equipo, debido a la coyuntura de apertura y a las evidencias de la crisis financiera a finales de la década.

En cuanto a la productividad en el sector industrial, el aporte por trabajador ha crecido continuamente, con un $1,7 \%$ anual (ver figura 1 ).

Figura 1. Productividad industrial colombiana expresada en millones de pesos por trabajador desde 1994

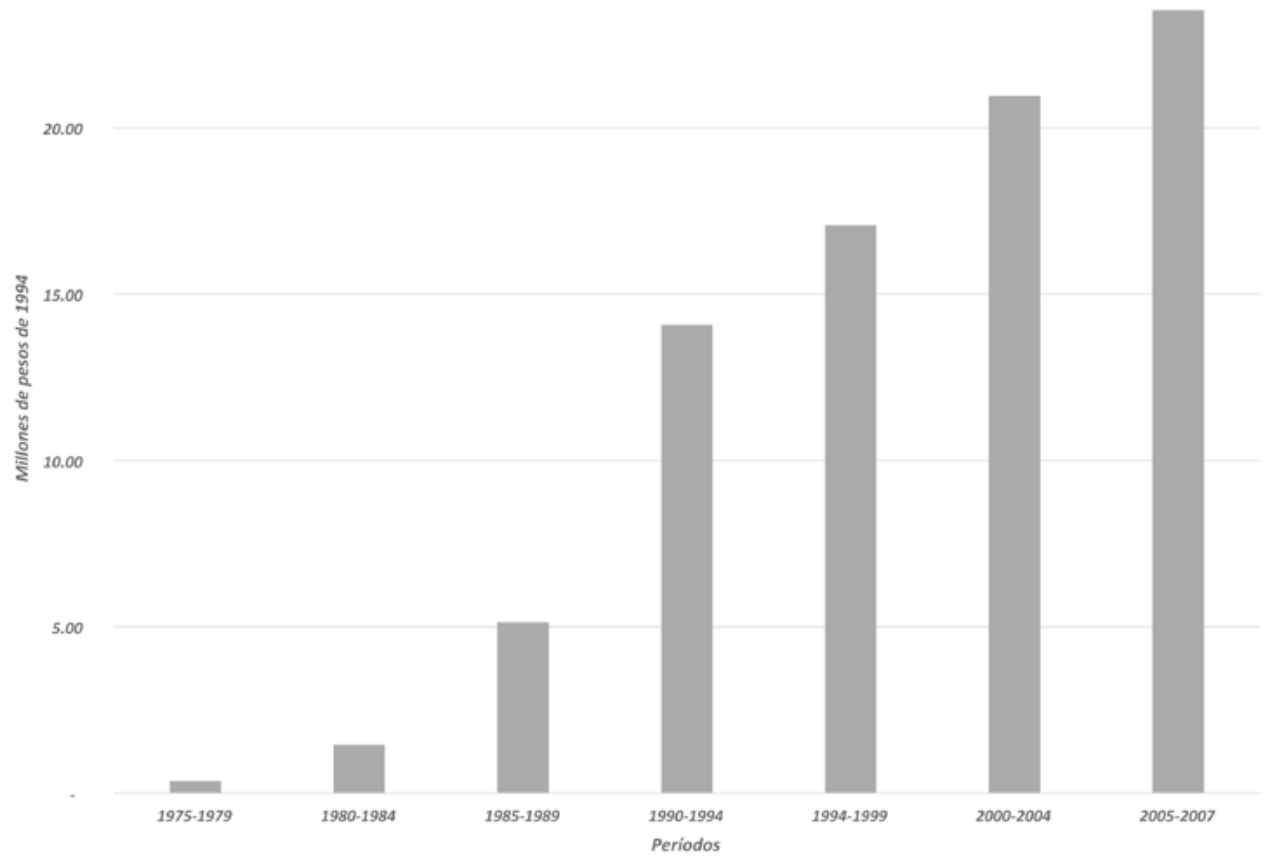

Fuente: DANE. Cálculos de los autores con información de las Cuentas Económicas Nacionales 1975-2007, Series empalmadas y Estadísticas del Mercado Laboral Colombiano (2014).

El problema del proceso de desindustrialización radica en que no se ha desarrollado una trasformación productiva en el sector industrial que vaya a la par con el sector servicios en la economía, debido a que el sector terciario presenta problemas de intermediación entre la industria, por ejemplo, comercio, servicios y desarrollo técnico. Así mismo, la industria tiene diferentes problemáticas que obedecen a varios factores, como el reflejo de una limitada estructura y modernización productiva, las deficiencias en cuanto al incremento de la acumulación de capital y el estancamiento en el patrón de la diversificación productiva. 
Figura 2. Cálculo de la relación de participación industrial PIB

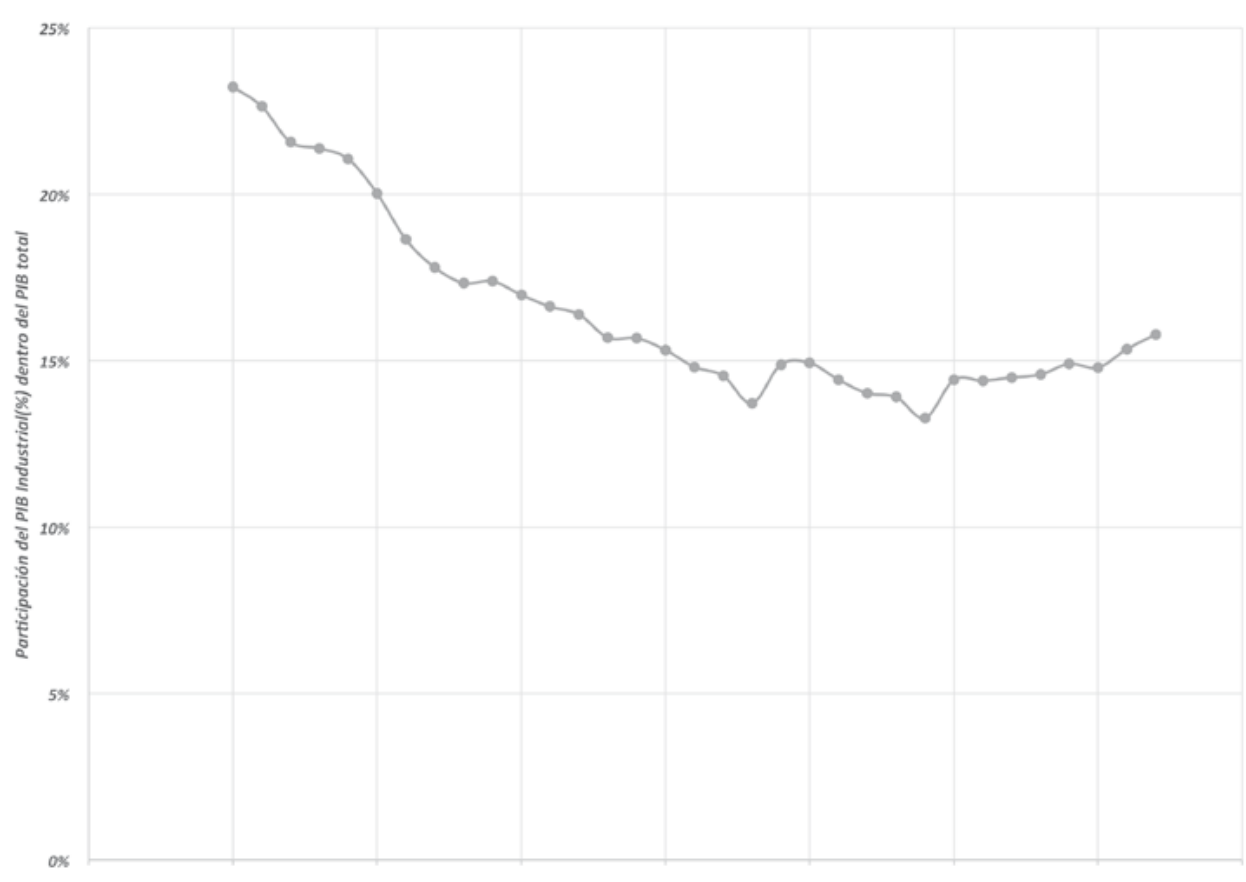

Fuente: DANE. Cálculos de los autores con información de las Cuentas Económicas Nacionales 1975 -2007. Series Empalmadas (2014)

Se puede observar en la figura 2 que, desde hace 20 años, desde la apertura económica, el sector industrial ha perdido participación acelerada en el PIB; según la gráfica, Colombia se ha venido desindustrializando desde los años setenta, pero este fenómeno ha sido más profundo desde la apertura económica.

Restrepo, Correa, Cadavid y Aguilar (2011) manifiestan que el proceso de internacionalización de la economía colombiana en los años noventa afectó a muchas empresas del sector industrial y las llevó hasta la quiebra, pero existen otras compañías de este mismo sector que han logrado sobresalir, sobrepasar los obstáculos que la apertura económica ha dejado y ser líderes pioneras tanto a nivel local como internacional gracias a sus esfuerzos invertidos en productividad e innovación. Un ejemplo claro de estas empresas es la conocida Industrias HACEB, compañía ubicada en Copacabana, en el norte de Medellín, dedicada a la producción, distribución y comercialización de electrodomésticos de manufactura de refrigeración y calefacción doméstica.

Esta empresa nació en 1940 gracias a su fundador José M. Acevedo, y sus productos se comercializan en gran parte de Latinoamérica y Estados Unidos, por ejemplo, en Ecuador, Venezuela, Costa Rica, Panamá y México. Desde su nacimiento, Industrias HACEB S. A. ha intentado siempre que la prioridad sea el cliente, y esto es pieza clave 
para el crecimiento de la empresa; otro pilar importante por el que optó es siempre estar a la vanguardia y crecer en cuanto a desarrollo tecnológico y economía de la innovación (Villamil, 2012).

Para ello, Industrias HACEB S. A. implementó cinco etapas, en las cuales se basó para crecer como empresa y ser pionera en el sector:

- Gonfiabilidad: Durante los primeros 35 años, el desarrollo tecnológico se enfocó en manufacturas de productos duraderos y de alta calidad.

- Funcionalidad: Con el negocio de refrigeración se introdujeron cambios en los productos, materiales y procesos.

- Seguridad: La producción de gas doméstico trajo, además, procesos más cortos y precisos.

- Modernización: A comienzos del siglo se alcanzaron los mayores niveles de producción de las plantas.

- Globalización: La planta de refrigeración más moderna en Latinoamérica, que entró en funcionamiento en 2012 y que cumple con todos los parámetros establecidos en el tema de eficiencia energética y cuidado del medio ambiente, implica una tecnología y una cultura de talla mundial (Restrepo et al, 2011).

La productividad y la competitividad han hecho que la compañía tenga un excelente desempeño en el mercado. Siempre con miras hacia el futuro, la empresa ha logrado complementar líneas de producto, introducir otras nuevas, ganarse mercado en Colombia y en el mundo y ser una de las empresas más importantes. Frente a los obstáculos que ha tenido la industria electrodoméstica, por ejemplo, la llegada de competidores extranjeros al país, se ha desenvuelto eficientemente en el mercado y con una prospectiva amplia hacia los escenarios futuros, es por ello que la compañía genera cinco propósitos, siempre enfocados en el cliente (Restrepo et al, 2011).

El primer y mayor propósito en cuanto a su focalización de mercado consiste en que los clientes tengan una experiencia satisfactoria con sus productos y que les genere confianza. Los otros cuatro objetivos son los siguientes: 1) que los trabajadores se sientan personas líderes y apasionados, 2) inspiración en el servicio, 3) crear valor agregado con responsabilidad social y 4) desarrollar equipos de alto rendimiento, con enfoques por procesos.

Teniendo en cuenta lo anterior, se pueden mensionar a Coltejer e Industrias HACEB como casos representativos, estos son dos de los casos más representativos de la industria colombiana. en donde Para el caso de es Coltejer, debido a la internacionalización de la economía tiene serios problemas estructurales en su forma de operar, hasta tal 
punto de que existan amenazas de que desaparezca si no se hacen cambios sustanciales en cuanto a productividad, competitividad e innovación. Entre tanto, Por otro lado, se puede ver la empresa Industrias HACEB, es una empresa que frente a los obstáculos generados por la apertura económica ha logrado sobrepasarlos con estrategias con miras hacia el futuro, con el eje central hacia el cliente y con grandes crecimientos de expansión. Como estos casos existen muchos en Colombia; algunas empresas han tomado la estrategia de relocalizarse o desmontarse verticalmente para poder ahorrar costos y gastos y ser más competitivas con miras a lograr una mejor participación en el mercado a nivel nacional e internacional.

\section{Conclusiones}

Se puede inferir que el fenómeno de la desindustrialización se presenta a nivel mundial y que se trata de un proceso espontáneo y sistemático que han sufrido todas las economías de mercado. En los países desarrollados se ha evidenciado una lenta desindustrialización debido a que recursos que antes poseía la industria se han ido desplazando a sectores más especializados y con alto contenido tecnológico; en estos países se presenta que el PIB per cápita supera los diez mil dólares, de lo cual se infiere que, a pesar de este fenómeno, la economía sigue siendo fuerte.

En contraposición se observa que los países en donde su diversificación exportadora consiste en materias primas, la industria ha tenido una disminución acelerada dentro de las economías desde la apertura económica y que, a diferencia de los países desarrollados, los recursos del sector industrial se están desplazando para el sector primario y también al sector terciario de la economía, recursos que se filtran dentro de estos sectores sin valor agregado ni productividad.

Estos múltiples factores se dieron debido a la apertura comercial y económica que evidenció Colombia finalizando los años ochenta con las políticas del entonces presidente Virgilio Barco, las cuales consolidaron este fenómeno bajo la presidencia de César Gaviria. Durante esa época, el Gobierno colombiano disminuyó progresivamente los aranceles hasta un $60 \%$ para textiles y confecciones, decisión que tuvo grandes repercusiones dentro de esta industria, por ejemplo: la eliminación de la producción algodonera; la balanza industrial se empezó a inclinar por el lado de importaciones y confecciones foráneas; cambio de la estructura interna de la cadena, que redujo la producción de hilo, fibras y textiles y aumentó la de confecciones, con un efecto negativo en los ingresos de los trabajadores del sector y que puso a depender la producción de prendas de vestir en Colombia del eslabón más débil de la cadena, que son las confecciones. 
Antes de los años noventa, Colombia tenía aproximadamente 300000 hectáreas de algodón, con una dependencia de un $90 \%$ en hilos y fibras. Después de la década de los noventa, ha habido una disminución considerable de los cultivos, llegando a 34000 hectáreas, es decir, un $10 \%$ de lo que antes se cultivaba.

En el caso de la balanza comercial, se ha visto que existe un aumento considerable de las importaciones textiles, que pasaron de 75 millones de pesos colombianos iniciando los años noventa a 1321 millones de pesos colombianos para 2007, de los cuales 788 corresponden a textiles y 333 a confecciones, lo cual ha aumentado la compra de textiles hacia el exterior hasta 17,5 veces, pasando de importar un $1 \%$ a $7 \%$.

Si bien la apertura económica de los años noventa ayudó a intensificar las relaciones comerciales con los diferentes países, especialmente con los de la región, se puede ver que el superávit en la balanza comercial para Colombia ha venido disminuyendo en los últimos años. En el caso de Venezuela, se observa que las exportaciones han aumentado considerablemente, pasando de USD 432 millones en 1990 a unos USD 1705 millones (textiles 408 y confecciones 1297) en el año 2007, con un crecimiento considerable del $80 \%$ menor que el de las importaciones; se pasó de un superávit de $83 \%$ a uno de $23 \%$, con riesgo de que siga disminuyendo hasta llegar al déficit.

El empleo ha sido el principal perjudicado, pues en 1990, cuando Colombia contaba con 32 millones de habitantes, el sector tenía 180000 empleados, y actualmente, con una población nacional de 45 millones - dato ofrecido en la Encuesta Integrada de Hogares publicada el 30 de junio de 2008- el sector tiene 105000 empleados ocupados.

En estos últimos 20 años se ha podido ver que el sector textil de las confecciones ha tenido un aumento significativo que se expresa en una disminución considerable de toda la cadena productiva de algodón, fibra e hilo, lo cual ha generado consecuencias e incentivos para las confecciones en lo que respecta a importar más productos textiles.

Aparte de todos estos problemas que se han visto, también existe otro relacionado con los términos de intercambio, pues el peso colombiano se ha venido apreciando considerablemente en comparación con el dólar de Estados Unidos, y esto implica que se importe más de lo que se exporte.

El Gobierno colombiano y las industrias textiles tiene un gran reto para el mantenimiento del sector. Se debe crear una política económica en la que los tratados de libre comercio con los diferentes países no dañen ni hundan el sector industrial textil. Así mismo, se deben crear políticas de competitividad y productividad para que esta industria, que está en déficit, tenga mejores prospectivas para su desarrollo hacia una internacionalización de la economía (DANE, 2014). 
El boom minero-energético en estos últimos 20 años por parte de Colombia ha hecho que la economía nacional dependa fuertemente de las exportaciones excesivas de hidrocarburos y minería. Debido a este comportamiento, se afirma que existen evidencias y síntomas del fenómeno de la enfermedad holandesa dentro de la economía nacional. Este fenómeno afecta directamente la industria, ya que, al entrar mucha moneda extranjera, se aprecia la moneda local, lo cual afecta las exportaciones, principalmente las del sector industrial.

Por otra parte, se puede ver que, debido a la coyuntura, el comportamiento de muchos empresarios ha sido invertir en productos foráneos para comercializarlos dentro de la economía nacional; por ende, se puede decir que muchas partes en donde se desarrolló la industria en Colombia se han ido volviendo más hacia el sector del comercio, un ejemplo de ello es la ciudad de Medellín.

La variable que más ha sido afectada en el ámbito económico dentro del sector industrial ha sido principalmente el empleo, ya que en los últimos 30 años ha disminuido drásticamente; causante de ello es la baja productividad y competitividad dentro de este sector, que ha dejado como consecuencias el desempleo y el empleo informal.

Si bien se ha hecho una investigación de carácter descriptivo, es importante establecer que el tema del fenómeno de la desindustrialización en Colombia es amplio y que abarca toda clase de características y variables que tienen que ver con el ámbito económico, por ende, cabe resaltar que en investigaciones futuras han de tenerse en cuenta más metodologías cuantitativas, con el fin de tener un alcance más claro y objetivo de la investigación.

También vale la pena estudiar cómo ha sido el comportamiento de este fenómeno en cada uno de las ciudades donde principalmente se ha desarrollado la industria en Colombia. Existen pocos estudios acerca de este tema y es importante abordarlos de manera particular, por ejemplo, el caso del proceso de desindustrialización de Medellín desde la apertura económica.

\section{Referencias}

Carranza, J. E. y Moreno, S. (2013). Tamaño y estructura vertical de la cadena de producción industrial desde 1990 (Serie Borradores de Economía n.o 751). Bogotá: Editorial Banco de la República. Recuperado de goo.gl/qoGzqq

Clavijo, S., Vera, A., Fandiño, A. (2012). La desindustrialización en Colombia. Análisis cuantitativo de sus determinantes. Bogotá Colombia: ANIF. Recuperado de goo.gl/x9y98P 
DANE. (2014). Estadísticas de participación industrial en el PIB. Recuperado de http://www. dane.gov.co/

Echavarría, J. y Villamizar, M. (2006). El proceso colombiano de desindustrialización (Serie Borradores de Economía n.o 361). Bogotá: Editorial Banco de la República. Recuperado de goo.gl/9fZcs9

Fedesarrollo. (2011). http://www.fedesarrollo.org.co/

FitzGerald, V. (1998). La CEPAL y la teoría de la industrialización. Revista de la CEPAL, n.o Extraordinario, 47-61. Recuperado de goo.gl/oijtlL

Galvis, L. A. (Ed.). (2014). Economía de las grandes ciudades en Colombia: seis estudios de casos (Colección de Economía Regional). Bogotá: Editorial Banco de la República. Recuperado de goo.gl/5nNykh

NU Naciones Unidas Nueva York y UNIDO United Nations Industrial Development Organization Viena. (1985). Industry in the 1980s: structural change and interdependence. Nueva York: Naciones Unidas, p. 228. Industrial development survey.

Ocampo, J., Bernal, J., Avella , M., \& Errázuriz, M. (1987). La Consolidación del Capitalismo Moderno (1945 -1986). En J. Ocampo, História Económica de Colombia (Guarta ed.). Bogotá: Siglo XXI Editores. Obtenido de http://www.banrepcultural. org/blaavirtual/economia/histecon/histecon8a.htm

Restrepo, L. F., Correa, Ó., Cadavid, A. y Aguilar, J. (2011). Haceb 70 años de liderazgo: el futuro nos conecta. Medellín: Panamericana Formas e Impresos

Sanabria- Gómez, S. (2007). Tres décadas de desindustrialización en Colombia. Apuntes del Cenes, 16. Recuperado de goo.gl/BkxNWD

Steiner, R. (2011). ¿Se está "des-industrializando" Colombia? Debates de Coyuntura Económica y Social, Fedesarrollo, 87, 15-19. Recuperado de goo.gl/vCtAM2

Villamil, L. (11 de julio de 2012). Un legado de US\$25 millones en innovación. El Espectador. Recuperado de goo.gl/QRW2z2 